\title{
Registro prospectivo del stent liberador de sirolimus con polímero estable de fluoroacrilato Angiolite: estudio EPIC02 - RANGO
}

\author{
Armando Pérez de Prado ${ }^{a}{ }^{*}$, Raymundo Ocaranza-Sánchez ${ }^{b}$ Fernando Lozano Ruiz-Poveda ${ }^{c}$, \\ José Moreu Burgos ${ }^{\mathrm{d}}$, Raquel Álvarez Ramose, Alberto Rodrigues ${ }^{\mathrm{f}}$, Luis Fernández González ${ }^{g}$, \\ Pablo Aguar ${ }^{\mathrm{h}}$, Bruno García del Blanco ${ }^{\mathrm{i}}$, Eduardo Pinari, Vicente Peral ${ }^{\mathrm{k}}$, Fermín Sainz Laso ${ }^{\mathrm{l}}$, \\ José Ramón Rumoroso ${ }^{\mathrm{m}}$, Alfonso Torres ${ }^{\mathrm{n}}$, Manel Sabaté ${ }^{\circ, \mathrm{p}}$ y Ramiro Trillo Nouche ${ }^{\mathrm{q}}$
}

a Servicio de Cardiología, Hospital Universitario de León, León, España

b Servicio de Cardiología, Hospital Lucus Augusti, Lugo, España

' Servicio de Cardiología, Hospital General Universitario Ciudad Real, Ciudad Real, España

d Servicio de Cardiología, Hospital Virgen de la Salud, Toledo, España

e Servicio de Cardiología, Hospital Universitario de La Fe, Valencia, España

${ }^{\mathrm{f}}$ Servicio de Cardiología, Hospital de Vila Nova, Gaia, Portugal

g Servicio de Cardiología, Hospital Cruces, Bilbao, España

h Servicio de Cardiología, Hospital Dr. Peset, Valencia, España

i Servicio de Cardiología, Hospital Vall D'Hebron, Barcelona, España

j Servicio de Cardiología, Hospital Virgen de la Arrixaca, El Palmar, Murcia, España

k Servicio de Cardiología, Hospital Son Espases, Palma de Mallorca, España

${ }^{1}$ Servicio de Cardiología, Hospital Marqués de Valdecilla, Santander, España

m Servicio de Cardiología, Hospital Galdakao-Usansolo, Bilbao, España

n Servicio de Cardiología, Hospital Universitario de Araba, Vitoria, Álava, España

- Servicio de Cardiología, Hospital Clínic, IDIBAPS, Barcelona, España

p Centro de Investigación Biomédica en Red de Enfermedades Cardiovasculares (CIBERCV), CIBERCV CB16/11/00411, España

q Servicio de Cardiología, Hospital Universitario de Santiago de Compostela, A Coruña, España

\section{VÉASE CONTENIDO RELACIONADO:}

https://doi.org/10.24875/RECIC.M21000226

\section{RESUMEN}

Introducción y objetivos: Para confirmar los resultados observados en análisis preclínicos y clínicos del stent liberador de sirolimus Angiolite se diseñó el registro observacional de vida real RANGO.

Métodos: El registro prospectivo multicéntrico incluyó pacientes con distintas indicaciones clínicas que recibieron al menos 1 stent Angiolite para tratar su enfermedad coronaria y que dieron su consentimiento informado. El objetivo primario fue la incidencia de fracaso del tratamiento de la lesión (FTL) a 6, 12 y 24 meses, definido como muerte de causa cardiaca, infarto de miocardio en relación con el vaso tratado o nueva revascularización de la lesión tratada. Los objetivos secundarios fueron los componentes individuales del objetivo primario y las incidencias de eventos cardiacos mayores (MACE) y de trombosis del stent. Se presentan los resultados del registro RANGO a 2 años en la población global, en los pacientes que recibieron stent Angiolite y en 2 subgrupos predefinidos de diabéticos y vasos pequeños $(\leq 2,5 \mathrm{~mm})$.

Resultados: Se seleccionaron 646 pacientes (426 solo recibieron stents Angiolite) con un perfil de riesgo elevado: infarto previo $(18,4 \%)$, revascularización coronaria previa $(23,4 \%)$, presentación clínica como infarto agudo con elevación del segmento ST $(23,1 \%)$ y enfermedad multivaso $(47,8 \%)$. A los 2 años, la incidencia de FTL en el grupo global fue del 3,4\%, la de MACE fue del 9,6\% y la de trombosis del stent fue del 0,9\%. En el grupo tratado solo con stents Angiolite, los resultados fueron similares (FTL 3,1\%, MACE $8,0 \%$ y trombosis $0,7 \%$ ). Los resultados no fueron significativamente diferentes en los diabéticos (FTL 3,0\%, MACE 14,1\% y trombosis $1,0 \%$ ) y en los pacientes con vasos pequeños (FTL 4,3\%, MACE $12,1 \%$ y trombosis $0 \%$ ).

Conclusiones: Los resultados del registro observacional RANGO a los 2 años en población de vida real con perfil de riesgo elevado confirman los excelentes resultados del stent Angiolite observados en estudios previos. Se plantea un seguimiento clínico a 5 años para descartar eventos muy tardíos.

Palabras clave: Stent liberador de sirolimus. Fluoropolímero estable. Estudio observacional. Eficacia. Seguridad. Trombosis del stent.

\footnotetext{
* Autor para correspondencia: Servicio de Cardiología, Hospital Universitario de León, Altos de Nava s/n, 24008 León, España. Correo electrónico: aperez@fundacionepic.org (A. Pérez de Prado).
} 


\section{Real-world registry of the durable Angiolite fluoroacrylate polymer-based sirolimus-eluting stent: the EPIC02 - the RANGO study}

\section{ABSTRACT}

Introduction and objectives: After the positive pre-clinical and clinical results with Angiolite, a cobalt-chromium sirolimus-eluting stent, we decided to analyze its performance in a non-selected, real-world population: the RANGO registry.

Methods: We conducted an observational, prospective, multicenter registry of patients with different clinical indications. All consecutive patients treated with percutaneous coronary intervention with, at least, 1 Angiolite stent and who gave their informed consent were included. The registry primary endpoint was the occurrence of target lesion failure (TLF) at 6,12 , and 24 months defined as cardiovascular death, myocardial infarction (MI) related to target vessel, and clinically driven target lesion revascularization. The secondary endpoints were the individual components of the primary endpoint, major adverse cardiovascular events (MACE: all-cause mortality, any MI, or any revascularization), and stent thrombosis. We describe the 2-year clinical results of the RANGO study in the entire population, in those who only received Angiolite stents, and in 2 predefined subgroups: diabetics and patients with small-vessels $(\leq 2.5 \mathrm{~mm})$.

Results: 646 patients (426 of them only received Angiolite stents) with a high-risk profile were recruited: prevalence of previous MI (18.4\%), previous coronary revascularization (23.4\%), clinical presentation as ST-segment elevation MI $(23.1 \%)$, and multivessel disease $(47.8 \%)$. At the 2-year follow-up, the rates of TLF, MACE, and stent thrombosis were $3.4 \%, 9.6 \%$, and $0.9 \%$, respectively. Similar results were observed among patients treated with Angiolite stents only: TLF, 3.1\%; MACE, $8.0 \%$; thrombosis, $0.7 \%$. The rates were not significantly different for the diabetic (TLF, 3.0\%; MACE, $14.1 \%$; thrombosis, $1.0 \%$ ), and small-vessel subgroups (TLF, $4.3 \%$; MACE, $12.1 \%$; thrombosis, $0 \%$ ).

Conclusions: In conclusion, the results of this observational registry on the use of Angiolite in a real-world population, including a high-risk population, corroborate the excellent results observed in previous studies, up to a 2-year follow-up. An extended 5-year follow-up is planned to discard the occurrence of late events.

Keywords: Sirolimus-eluting-stent. Durable fluoropolymer. Observational study. Efficacy. Safety. Stent thrombosis.

\section{Abreviaturas}

FLD: fracaso de la lesión diana. ICP: intervención coronaria percutánea. MACE: eventos cardiovasculares adversos mayores. RLD: revascularización de la lesión diana. RVD: revascularización del vaso diana. SFA: stent farmacoactivo.

\section{INTRODUCCIÓN}

Los stents farmacoactivos (SFA) representan uno de los mayores avances en el tratamiento percutáneo de la enfermedad coronaria. Estos dispositivos vienen demostrando consistentemente incidencias más bajas de revascularización del vaso diana en diferentes situaciones clínicas convirtiéndose en el tratamiento preferido ${ }^{1}$. No obstante, el riesgo de trombosis del stent tardía y muy tardía surgió con los SFA de primera generación ${ }^{2}$ y, hasta la fecha, sigue siendo motivo de preocupación ${ }^{3}$. Este fenómeno se asocia a efectos secundarios al fármaco (que entorpecen la proliferación de nuevas células endoteliales), al polímero, a la plataforma del stent o a un compendio de todos ellos en la pared del vaso, lo que acarrea endotelización incompleta, reacciones inflamatorias persistentes y el desarrollo de neoaterosclerosis. Se han desarrollado nuevos SFA más eficaces para no tener que revascularizar que garantizan incidencias mucho más bajas de trombosis del stent, la manifestación clínica más temida de la curación subóptima del vaso. El stent Angiolite (iVascular, España) es un stent liberador de sirolimus fabricado en una aleación de cromo-cobalto y struts finos con un recubrimiento polimérico bioestable que consta de 3 capas: acrilato para garantizar la adhesión a la superficie de metal, acrilato fluorado cargado con sirolimus $\left(1,4 \mu \mathrm{g} / \mathrm{mm}^{2}\right)$ y una capa superior de acrilato fluorado para controlar la liberación del fármaco (> 75\% liberación durante el primer mes).

El stent Angiolite se probó inicialmente en un modelo preclínico con resultados muy prometedores ${ }^{4}$ con una respuesta antiproliferativa equivalente y un mejor patrón de curación que el stent XIENCE (Abbott Vascular, Estados Unidos). Después, el estudio ANCHOR, el primero de su clase realizado en humanos ${ }^{5}$ confirmó, mediante OCT, la potente inhibición de la hiperplasia neointimal: el stent Angiolite inhibió eficazmente la respuesta proliferativa (estenosis del área del vaso afectado, $4,4 \pm 11,3 \%$ ), la pérdida luminal intrastent a los 6 meses $(0,07 \pm 0,37 \mathrm{~mm})$ y tuvo una incidencia más baja de mala aposición de los struts $(1,1 \pm 6,2 \%)$. Por último, el estudio clínico aleatorizado ANGIOLITE ${ }^{6}$ comparó el stent Angiolite y el stent XIENCE en 223 pacientes (aleatorización 1:1). En este estudio, el objetivo primario, la pérdida de luz tardía intra-stent a los 6 meses no fue inferior en el grupo Angiolite $(0,04 \pm 0,39 \mathrm{~mm})$ comparado con el grupo XIENCE $(0,08 \pm 0,38 \mathrm{~mm})$. El stent ha recibido el marcado CE (Conformité Européenne) para su uso rutinario. Así pues, este registro prospectivo y observacional se diseñó para avalar los anteriores resultados en la práctica clínica habitual con indicaciones más amplias para su uso.

\section{MÉTODOS}

\section{Diseño del estudio}

El estudio EPIC02-RANGO se diseñó como un registro observacional, prospectivo, y multicéntrico de brazo único para evaluar el perfil de seguridad y eficacia del stent Angiolite en pacientes no seleccionados representativos de la práctica clínica habitual. El diseño del estudio lo aprobaron todos los investigadores, así como el patrocinador. Un comité de ética de referencia aprobó el protocolo del estudio, así como los formularios con los consentimientos informados; los comités de ética locales fueron informados de que este estudio se realizaría en sus respectivos centros de conformidad 
con la legislación nacional vigente. El estudio fue realizado y supervisado por una organización de investigación por contrato independiente. Los autores del presente manuscrito original llevaron a cabo, de manera independiente, el análisis final de los datos, interpretaron los resultados del estudio y redactaron el manuscrito original. El patrocinador fue informado tanto de las fases del estudio como de los resultados finales, pero no tuvo ninguna participación en los mismos.

\section{Selección de la población del estudio}

Para poder incluirse en el estudio, los sujetos debían cumplir los siguientes 3 criterios de inclusión: $\geq 18$ años, haber sido tratados de intervención coronaria percutánea (ICP) con, al menos, 1 stent Angiolite y haber sido convenientemente informados y firmado el correspondiente formulario de consentimiento informado.

A fin de garantizar una población del mundo real, se aplicaron criterios de exclusión que no fuesen restrictivos. Así, solo se excluyó del estudio a aquellos sujetos que cumplieran alguno de los siguientes criterios de exclusión: contraindicación para recibir terapia antiplaquetaria doble, situación de shock cardiogénico, pocas probabilidades de completar el seguimiento programado o una renuncia formal a participar en el estudio.

La ICP (predilatación, imagen intracoronaria, posdilatación, planificación y manejo final) se dejó a criterio del operador y fue indicativo del uso de stents en el mundo real. El tratamiento médico durante y después de la intervención, incluido el régimen antiplaquetario y la duración de este, también se ajustó a las prácticas locales estándar; no obstante, se sugirió a los investigadores que siguiesen las guías disponibles sobre el manejo de estos pacientes ${ }^{1,7}$.

\section{Objetivos del estudio}

El objetivo primario fue el fracaso de la lesión diana (FLD) a los 6, 12 y 24 meses, definida como muerte cardiovascular, infarto de miocardio del vaso diana o revascularización de la lesión diana por motivos clínicos.

Los objetivos secundarios fueron:

- El fracaso del vaso diana, definido como muerte cardiovascular, infarto de miocardio del vaso diana o revascularización del vaso diana.

- Los eventos cardiovasculares adversos mayores (MACE) definidos como mortalidad por cualquier causa, cualquier infarto de miocardio o cualquier revascularización del vaso diana.

- La trombosis del stent (definitiva o probable según los criterios del $\left.\mathrm{ARC}^{8}\right)$.

En todos los casos, el infarto de miocardio se refiere, solo, a infartos espontáneos. Se predefinieron 2 subgrupos: pacientes con diabetes y pacientes con stents Angiolite colocados en pequeños vasos (diámetro del stent $\leq 2,5 \mathrm{~mm}$ ).

\section{Cálculo del tamaño de la muestra}

Se realizó un análisis exploratorio que dio como resultado una población de 640 pacientes (con una pérdida estimada al seguimiento del 10\%). Este tamaño muestral arrojó un intervalo de confianza bilateral del $95 \%$ con una precisión del 1,75\% con incidencias de FLD del 4,86\%. Este valor se obtuvo de los datos publicados sobre diferentes stents contemporáneos $^{9-17}$ (tabla 1 del material adicional).

\section{Análisis poblacional primario}

El análisis primario del perfil de seguridad y eficacia tuvo en cuenta a todos los pacientes que recibieron el stent Angiolite salvo aquellos que retiraron su consentimiento informado. El análisis secundario se realizó en todos los pacientes incluidos en el estudio que recibieron, al menos, 1 stent Angiolite más otro stent diferente a excepción de aquellos que retiraron su consentimiento informado

\section{Comité de eventos clínicos}

Un comité independiente de monitorización de datos de seguridad revisó los datos de seguridad acumulados para salvaguardar el bienestar de los participantes. Todos los eventos fueron monitorizados en remoto por una organización de investigación por contrato. El comité de eventos clínicos revisó, adjudicó y clasificó todos los eventos adversos. Ninguno de los 5 miembros del comité de eventos clínicos estaba afiliado a ninguno de los centros que participaron en el estudio.

Se realizaron al azar 90 auditorías entre los pacientes (el 14\% de la población global) en 4 centros, incluidos los 3 principales centros reclutadores. El resultado de estas auditorías fue que 9 eventos no se habían registrado, la mayoría de los cuales correspondían a intervenciones programadas que precisaron hospitalización (cirugías no cardiovasculares y 2 casos de ICP programadas). Ninguno de los eventos asociados a estas auditorías se correspondió con los eventos clasificados como objetivos primarios o secundarios.

\section{Análisis estadístico descriptivo}

Las variables continuas se presentan usando la siguiente estadística descriptiva: $n$ (basada en el número de valores de datos registrados para cada parámetro), media, desviación estándar, intervalo de confianza del $95 \%$ para la media, mediana, rango intercuartil [Q1, Q3], máximo y mínimo. Las variables categóricas se presentan mediante la frecuencia y los porcentajes (basados en el número de valores de datos registrados para cada parámetro). En general, se proporcionan todos los datos discriminados por centro de estudio y sujeto.

\section{Métodos estadísticos}

Los resultados de las variables continuas se expresaron como media \pm desviación estándar. Las variables se compararon usando una prueba $t$ de Student independiente o la prueba de U de MannWhitney, según proceda. Las variables categóricas se expresaron como recuentos y porcentajes y se compararon usando la prueba de la $\mathrm{X}^{2}$ o la prueba exacta de Fisher. Las variables se compararon entre los pacientes tratados solo con el stent Angiolite y entre aquellos tratados con otros stents además del Angiolite. Las variables clínicas a los 6, 12 y 24 meses se expresaron como recuentos y porcentajes. Las curvas de riesgo tiempo hasta evento de interés se expresaron mediante cálculos de Kaplan-Meier.

Estos métodos se aplicaron en toda la cohorte y en los 2 subgrupos predefinidos, cuando fuese necesario: pacientes con diabetes y pacientes con lesiones en pequeños vasos (diámetros del stent $\leq 2,5 \mathrm{~mm}$ ).

Se empleó el software estadístico SAS versión 9.4 para todos los análisis estadísticos, listados, tabulaciones y figuras. 


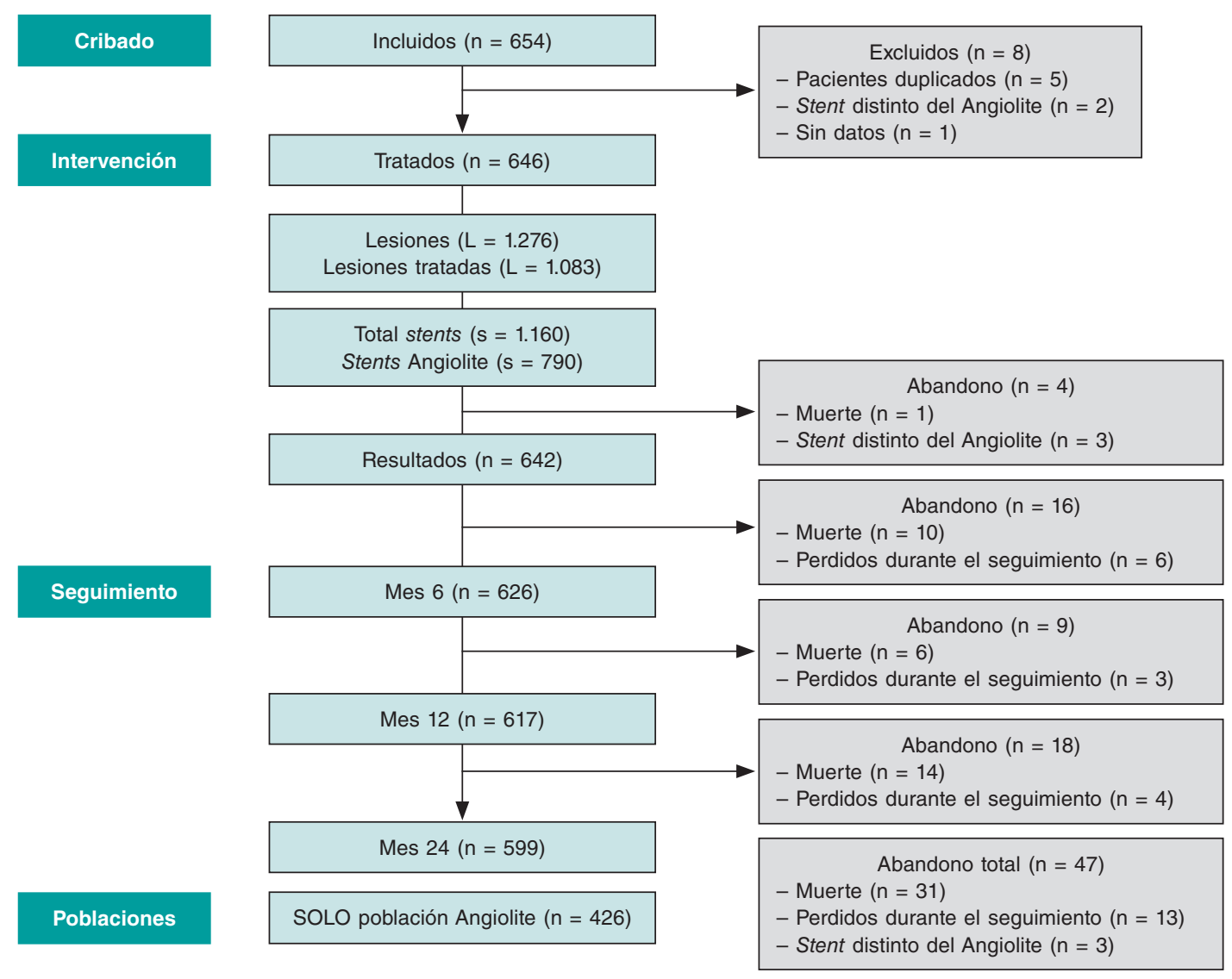

Figura 1. Diagrama de flujo del estudio.

\section{RESULTADOS}

Se inscribió a un total de 654 pacientes de 16 centros médicos universitarios de España y Portugal entre junio de 2017 y julio de 2018. Se excluyó a 8 pacientes por no cumplir los criterios de selección (2 en quienes no se intentó implantar el stent Angiolite, 5 duplicados con intervenciones programadas por etapas y 1 paciente del que no había datos disponibles). La población analizada consistió en 646 pacientes (figura 1); se trató a 426 pacientes solo con el stent Angiolite (análisis primario).

Las características basales y los datos clínicos se muestran en la tabla 1; las características angiográficas y de la intervención se muestran en la tabla 2. Cabe destacar que la población tiene un perfil de riesgo alto con una notable prevalencia del infarto de miocardio previo $(18,4 \%)$, la revascularización coronaria previa $(23,4 \%)$, la presentación clínica de infarto de miocardio con elevación del segmento ST $(23,1 \%)$ y la enfermedad multivaso $(47,9 \%)$.

La media \pm desviación estándar del número de lesiones por paciente fue $1,98 \pm 1,2$, el número medio de lesiones tratadas por paciente fue $1,68 \pm 0,9$ con un número medio de stents por paciente de 1,80 $\pm 1,1$. Estas cifras fueron mucho menores entre los pacientes tratados con el stent Angiolite y consistentes con los distintos perfiles de los pacientes. La tabla 3 resume las características y el tratamiento de cada lesión individual. Cabe destacar que los stents Angiolite se emplearon con mayor frecuencia para tratar la arteria asociada al infarto que los demás stents usados en nuestra población. Consecuentemente, las lesiones con trombo fueron más habituales en el grupo tratado solo con stents Angiolite si bien la calcificación grave fue más prevalente en el grupo global. Sobrevinieron complicaciones operatorias en 10 pacientes, en 7 de ellos asociadas a los stents Angiolite:
1 lesión imposible de cruzar, 1 perforación distal asociada a la guía de angioplastia, 1 fenómeno de falta de flujo miocárdico (no reflow) grave y 4 casos de disecciones, 2 de ellas tratadas con stents adicionales. Los índices de éxito tanto de la intervención como del implante del dispositivo fueron del 99,7 y 99,2\%, respectivamente. En escenarios anatómicos más complejos como, por ejemplo, lesiones con calcificación moderada/grave, los índices de éxito de la intervención y del implante del dispositivo siguieron siendo altos $(99,6$ y 99,3\%, respectivamente). Índices que llegaron al $100 \%$ en el subgrupo de lesiones en bifurcación o en el tronco común izquierdo.

Los seguimientos a 6 meses y 1 año fueron buenos, perdiéndose solo $9(1,4 \%)$ y $12(1,9 \%)$ pacientes durante el seguimiento, respectivamente. Tras 1 año de seguimiento, 368 pacientes $(59,6 \%)$ seguían a tratamiento antiplaquetario doble; una incidencia que cayó hasta el $15,5 \%$ al cabo de 2 años. Durante el periodo de seguimiento establecido (2 años para todos los pacientes), solo se perdieron 13 pacientes $(2 \%)$. En la población global, al cabo de 2 años, las incidencias de FLD, fracaso del vaso diana y MACE fueron del 3,4, 4,3 y 9,6\%, respectivamente. Dos de los 9 casos de FLD no se debieron a los stents Angiolite, sino a otros stents implantados. La incidencia de trombosis del stent definitiva/probable fue del 0,9\%; todos los pacientes estaban en tratamiento antiplaquetario doble cuando sobrevino este evento. Cabe destacar que durante la primera semana de seguimiento se detectaron 4 casos, 1 durante el primer mes y solo 1 caso de trombosis del stent al cabo de 6 meses (268 días). La tabla 4 y la figura 2 resumen la incidencia y el momento de ocurrencia de cada evento.

En el análisis poblacional primario (pacientes tratados solo con stents Angiolite) al cabo de 2 años, las incidencias de FLD, fracaso del vaso diana y MACE fueron del 3,1, 4,0 y 8,0\%, respectivamente. $\mathrm{La}$ incidencia de la trombosis del stent definitiva/probable fue del 
Tabla 1. Características basales y clínicas

\begin{tabular}{|c|c|c|}
\hline & $\begin{array}{l}\text { Total } \\
n=646\end{array}$ & $\begin{array}{l}\text { Solo población Angiolite } \\
n=426\end{array}$ \\
\hline Edad (años) & $66,41 \pm 11,93$ & $65,72 \pm 11,98$ \\
\hline Sexo varón & $495(76,6 \%)$ & $320(75,1 \%)$ \\
\hline \multicolumn{3}{|c|}{ Factores de riesgo cardiovascular e historia clínica } \\
\hline Hipertensión & $402(62,2 \%)$ & $254(59,6 \%)$ \\
\hline Dislipemia & $385(59,6 \%)$ & $251(58,9 \%)$ \\
\hline Diabetes mellitus* & $199(30,8 \%)$ & $119(27,9 \%)$ \\
\hline Fumador en la actualidad & $182(28,2 \%)$ & $127(29,8 \%)$ \\
\hline Fumador en el pasado & $28(4,3 \%)$ & $17(4,0 \%)$ \\
\hline Insuficiencia renal crónica & $46(7,1 \%)$ & $25(5,9 \%)$ \\
\hline Enfermedad vascular periférica* & $44(6,8 \%)$ & $23(5,4 \%)$ \\
\hline Infarto de miocardio previo & $119(18,4 \%)$ & $73(17,1 \%)$ \\
\hline $\begin{array}{l}\text { Cirugía de revascularización } \\
\text { coronaria previa }\end{array}$ & $20(3,1 \%)$ & $13(3,1 \%)$ \\
\hline ICP previa & $131(20,3 \%)$ & $78(18,3 \%)$ \\
\hline Fibrilación auricular & $34(5,3 \%)$ & $20(4,7 \%)$ \\
\hline Insuficiencia cardiaca & $46(7,1 \%)$ & $32(7,5 \%)$ \\
\hline Valvulopatía $\geq$ grado III & $16(2,5 \%)$ & $7(1,6 \%)$ \\
\hline \multicolumn{3}{|l|}{ Indicación para ICP } \\
\hline IAMSEST & $220(34,1 \%)$ & $141(33,1 \%)$ \\
\hline IAMCEST & $149(23,1 \%)$ & $112(26,3 \%)$ \\
\hline Angina estable & $120(18,6 \%)$ & $68(16,0 \%)$ \\
\hline $\begin{array}{l}\text { Angina inestable (biomarcadores } \\
\text { negativos) }\end{array}$ & $72(11,1 \%)$ & $51(12,0 \%)$ \\
\hline Isquemia miocárdica silente & $32(5,0 \%)$ & $19(4,5 \%)$ \\
\hline Otros & $53(8,2 \%)$ & $35(8,2 \%)$ \\
\hline
\end{tabular}

IAMCEST: infarto agudo de miocardio con elevación del segmento ST; IAMSEST: infarto agudo de miocardio sin elevación del segmento ST; ICP: intervención coronaria percutánea. * Diferencias significativas entre pacientes con solo el stent Angiolite y pacientes con cualquier otro stent además del Angiolite, $p<0,05$.

Los datos expresan n $(\%)$ o media \pm desviación estándar.

$0,7 \%$. No hubo ningún caso de trombosis del stent pasados los primeros 6 meses. La tabla 5 y la figura 3 resumen tanto la incidencia como el momento de ocurrencia de cada evento.

El análisis de subgrupo reveló los resultados a 2 años que fueron algo peores que los observados en la población global:

- El subgrupo diabético tenía unas incidencias de FLD, fracaso del vaso diana y MACE del 3,0, 4,5 y 14,1\%, respectivamente. La incidencia de trombosis del stent fue del 1,0\%: 2 casos entre 199 pacientes diabéticos; solo 1 de estos casos apareció en el análisis primario de pacientes tratados solo con el stent Angiolite. Los datos adicionales ofrecen una descripción de la incidencia de cada evento (tabla 2 del material adicional).

- Los pacientes con stents implantados en pequeños vasos $(\leq 2,5 \mathrm{~mm})$ revelaron incidencias de FLD, fracaso del vaso diana y MACE
Tabla 2. Características angiográficas y de la intervención

\begin{tabular}{|c|c|c|}
\hline & $\begin{array}{l}\text { Total } \\
n=646\end{array}$ & $\begin{array}{l}\text { Solo población Angiolite } \\
n=426\end{array}$ \\
\hline \multicolumn{3}{|l|}{ Coronariografía } \\
\hline Abordaje radial & $585(90,6 \%)$ & $396(93,0 \%)$ \\
\hline \multicolumn{3}{|l|}{ Extensión de la enfermedad } \\
\hline \multicolumn{3}{|l|}{$N^{o}$ de vasos enfermos* } \\
\hline 1 & $337(52,2 \%)$ & $289(67,8 \%)$ \\
\hline 2 & $198(30,7 \%)$ & $92(21,6 \%)$ \\
\hline 3 & $111(17,1 \%)$ & $45(10,6 \%)$ \\
\hline Tronco común izquierdo* & $29(4,5 \%)$ & $12(2,8 \%)$ \\
\hline Enfermedad de DA proximal & $179(27,7 \%)$ & $110(25,8 \%)$ \\
\hline Enfermedad difusa* & $128(19,8 \%)$ & $63(14,8 \%)$ \\
\hline$N^{o}$ de lesiones por paciente ${ }^{*}$ & $1,98 \pm 1,24$ & $1,51 \pm 0,90$ \\
\hline$N^{\circ}$ de lesiones tratadas por paciente & $1,68 \pm 0,95$ & $1,25 \pm 0,53$ \\
\hline$N^{o}$ de stents por paciente* & $1,80 \pm 1,11$ & $1,24 \pm 0,55$ \\
\hline \multicolumn{3}{|l|}{ Intervención índice } \\
\hline \multicolumn{3}{|l|}{ Revascularización } \\
\hline Completa & $489(75,7 \%)$ & $331(77,7 \%)$ \\
\hline Funcional & $84(13,0 \%)$ & $51(12,0 \%)$ \\
\hline \multicolumn{3}{|l|}{ Técnicas de imagen intravascular } \\
\hline IVUS & $15(2,3 \%)$ & $5(1,2 \%)$ \\
\hline ОСТ & $12(1,9 \%)$ & $7(1,6 \%)$ \\
\hline Revascularización por etapas* & $85(13,2 \%)$ & $26(6,1 \%)$ \\
\hline
\end{tabular}

DA: arteria descendente anterior izquierda; IVUS: ecografía intravascular; OCT: tomografía de coherencia óptica.

* Diferencias significativas entre pacientes con solo el stent Angiolite y pacientes con cualquier otro stent además del Angiolite, $\mathrm{p}<0,05$.

Los datos expresan $\mathrm{n}(\%)$ o media \pm desviación estándar.

del $4,3,6,0$ y $12,1 \%$, respectivamente. No hubo ningún episodio de trombosis del stent. Los datos adicionales ofrecen una descripción de la incidencia de cada evento (tabla 3 del material adicional).

\section{DISCUSIÓN}

Los resultados del presente registro del mundo real del stent coronario Angiolite revelan un perfil de seguridad y eficacia sobresaliente tal y como reflejaban los resultados del primer estudio realizado en humanos, $\mathrm{ANCHOR}^{5}$, y el ensayo clínico aleatorizado ANGIOLITE 6 , comparándose con el stent XIENCE. El perfil clínico revela una población de riesgo relativamente alto con una prevalencia de diabetes mellitus del $30,8 \%$, del $17,6 \%$ con anticoagulación oral, del $18,4 \%$ en pacientes diagnosticados de infarto de miocardio previo y del $23,4 \%$ en pacientes con revascularización coronaria previa. También se halló una alta tasa de incidencia de enfermedad coronaria compleja en la población reclutada: se diagnosticó enfermedad multivaso significativa en el $47,8 \%$, tronco común izquierdo comprometido en el $4,5 \%$ y enfermedad coronaria difusa en el 19,8\% de los pacientes. En consecuencia, el número 
Tabla 3. Características y tratamiento de cada lesión

\begin{tabular}{|c|c|c|}
\hline & $\begin{array}{l}\text { Total } \\
\mathrm{L}=1.083(84,9 \% \\
\text { de todas } \\
\text { las lesiones) }\end{array}$ & $\begin{array}{l}\text { Solo población } \\
\text { Angiolite } \\
L=531 \text { (82,5\% } \\
\text { de todas } \\
\text { las lesiones) }\end{array}$ \\
\hline \multicolumn{3}{|l|}{ Vaso } \\
\hline $\begin{array}{l}\text { Territorio de la descendente anterior } \\
\text { izquierda }\end{array}$ & $459(42,4 \%)$ & $236(44,4 \%)$ \\
\hline Territorio de la coronaria derecha & $327(30,2 \%)$ & $172(32,4 \%)$ \\
\hline Territorio de la circunfleja izquierda & $273(24,9 \%)$ & $112(21,2 \%)$ \\
\hline Tronco común izquierdo & $19(1,8 \%)$ & $5(0,9 \%)$ \\
\hline Otro & $5(0,7 \%)$ & $6(1,1 \%)$ \\
\hline \multicolumn{3}{|l|}{ Clasificación $A H A / A C C^{*}$} \\
\hline A & $95(8,8 \%)$ & $68(12,8 \%)$ \\
\hline B1 & $355(32,8 \%)$ & $193(36,3 \%)$ \\
\hline B2 & $429(39,6 \%)$ & $185(34,8 \%)$ \\
\hline C & $204(18,8 \%)$ & $85(16,0 \%)$ \\
\hline \multicolumn{3}{|l|}{ Características de la lesión } \\
\hline Trombo* & $145(13,4 \%)$ & $91(17,1 \%)$ \\
\hline Stent en la arteria asociada al infarto* & $366(33,8 \%)$ & $249(46,9 \%)$ \\
\hline Calcificación grave* & $85(7,8 \%)$ & $22(4,1 \%)$ \\
\hline Lesión reestenótica tratada & $37(3,4 \%)$ & $22(4,1 \%)$ \\
\hline Oclusión coronaria total crónica & $37(3,4 \%)$ & $20(3,8 \%)$ \\
\hline Lesión en bifurcación & $108(10,0 \%)$ & $47(8,9 \%)$ \\
\hline Tortuosidad grave & $142(13,1 \%)$ & $62(11,7 \%)$ \\
\hline Diámetro del vaso $(\mathrm{mm})$ & $2,91 \pm 0,55$ & $2,91 \pm 0,53$ \\
\hline Longitud de la lesión $(\mathrm{mm})^{*}$ & $19,47 \pm 9,80$ & $17,56 \pm 8,26$ \\
\hline Predilatación* & $786(72,6 \%)$ & $363(68,4 \%)$ \\
\hline Scoring balloon & $45(4,2 \%)$ & $11(2,1 \%)$ \\
\hline Scoring balloon & $28(2,6 \%)$ & $8(1,5 \%)$ \\
\hline Aterectomía rotacional & $27(2,5 \%)$ & $9(1,7 \%)$ \\
\hline Trombectomía* & $75(6,9 \%)$ & $48(9,0 \%)$ \\
\hline Stents implantados & $S=1160$ & $S=529$ \\
\hline$N^{o}$ de stents por lesión & $1,07 \pm 0,45$ & $1,00 \pm 0,35$ \\
\hline \multicolumn{3}{|l|}{ Características del stent* } \\
\hline Tipo $=$ Stent Angiolite & $784(67,6 \%)$ & $529(100,0 \%)$ \\
\hline Diámetro del stent $(\mathrm{mm})$ & $2,99 \pm 0,51$ & $2,99 \pm 0,46$ \\
\hline Longitud del stent $(\mathrm{mm})$ & $21,38 \pm 8,51$ & $20,34 \pm 7,03$ \\
\hline Presión máxima (atm) & $14,61 \pm 2,48$ & $14,69 \pm 2,46$ \\
\hline Stent cruzando la lesión al 1er intento & $1.067(98,5 \%)$ & $527(99,2 \%)$ \\
\hline Lesiones en bifurcación & $104(96,3 \%)$ & $45(95,7 \%)$ \\
\hline Calcificación moderada o grave & $268(97,1 \%)$ & $75(97,4 \%)$ \\
\hline Tronco común izquierdo & $19(100 \%)$ & $5(100 \%)$ \\
\hline Posdilatación & $284(26,2 \%)$ & $149(28,1 \%)$ \\
\hline Diámetro del balón (mm) & $3,24 \pm 0,62$ & $3,25 \pm 0,53$ \\
\hline Tipo de balón, no distensible & $186(67,4 \%)$ & $112(76,7 \%)$ \\
\hline
\end{tabular}

ACC: American College of Cardiology, AHA: American Heart Association; L: lesiones; S: stents.

* Diferencias significativas entre pacientes con solo el stent Angiolite y pacientes con cualquier otro stent además del Angiolite, $\mathrm{p}<0,05$.

Los datos expresan n (\%) o media \pm desviación estándar.
Tabla 4. Resultados en la población general

\begin{tabular}{|c|c|c|c|}
\hline Población total $(n=646)$ & $\begin{array}{l}6 \text { meses de } \\
\text { seguimiento }\end{array}$ & $\begin{array}{l}1 \text { año de } \\
\text { seguimiento }\end{array}$ & $\begin{array}{l}2 \text { años de } \\
\text { seguimiento }\end{array}$ \\
\hline Mortalidad & $11(1,7 \%)$ & $17(2,6 \%)$ & $31(4,8 \%)$ \\
\hline Mortalidad cardiovascular & $6(0,9 \%)$ & $8(1,2 \%)$ & $11(1,7 \%)$ \\
\hline Infarto de miocardio & $11(1,7 \%)$ & $16(2,5 \%)$ & $20(3,1 \%)$ \\
\hline Infarto de miocardio del vaso diana & $6(0,9 \%)$ & $8(1,2 \%)$ & $8(1,2 \%)$ \\
\hline $\begin{array}{l}\text { Trombosis definitiva/probable } \\
\text { del dispositivo }\end{array}$ & $5(0,8 \%)$ & $6(0,9 \%)$ & $6(0,9 \%)$ \\
\hline Revascularización & $13(2,0 \%)$ & $22(3,4 \%)$ & $32(5,0 \%)$ \\
\hline $\begin{array}{l}\text { Revascularización de la lesión } \\
\text { diana }\end{array}$ & $6(0,9 \%)$ & $8(1,2 \%)$ & $9(1,4 \%)$ \\
\hline Revascularización del vaso diana & $7(1,1 \%)$ & $11(1,7 \%)$ & $15(2,3 \%)$ \\
\hline $\begin{array}{l}\text { Revascularización del vaso } \\
\text { no diana }\end{array}$ & $6(0,9 \%)$ & $11(1,7 \%)$ & $17(2,6 \%)$ \\
\hline Fracaso de la lesión diana ${ }^{a}$ & $13(2,0 \%)$ & $18(2,8 \%)$ & $22(3,4 \%)$ \\
\hline Fracaso del vaso diana ${ }^{b}$ & $14(2,2 \%)$ & $21(3,3 \%)$ & $28(4,3 \%)$ \\
\hline$M^{\prime} A C E^{c}$ & $25(3,9 \%)$ & $41(6,3 \%)$ & $62(9,6 \%)$ \\
\hline
\end{tabular}

MACE: eventos cardiovasculares adversos mayores.

a Fracaso de la lesión diana definido como muerte cardiovascular, infarto de miocardio del vaso diana y revascularización de la lesión diana por motivos clínicos.

${ }^{\mathrm{b}}$ Fracaso del vaso diana definido como muerte cardiovascular, infarto de miocardio del vaso diana y revascularización del vaso diana.

${ }^{\circ}$ MACE definidos como mortalidad por cualquier causa, cualquier infarto y cualquier revascularización.

medio de lesiones significativas $(1,98 \pm 1,24)$, lesiones tratadas $(1,68$ $\pm 0,95)$ y stents implantados por paciente $(1,8 \pm 1,11)$ fue relativamente alto. El contexto de infarto agudo de miocardio con elevación del segmento ST de la ICP también revela, en la cuarta parte de los casos, la naturaleza de mundo real y pacientes no seleccionados del estudio.

El registro se diseñó para incluir a todos los pacientes en quienes se fuera a implantar el stent Angiolilte. Así, se pueden distinguir 2 poblaciones distintas: aquellos en quienes SOLO se iba a implantar el stent Angiolite (análisis primario) y aquellos que recibieron diferente stents para el tratamiento de otras lesiones además del stent Angiolite (análisis secundario). Estas poblaciones presentan algunas diferencias importantes: aquellos pacientes que SOLO recibieron el stent Angiolite eran más propensos a tener 1 solo vaso enfermo, menos lesiones significativas, menos lesiones tratadas y menos stents implantados. Lógicamente, esta población de una menor carga aterosclerótica presentaba menos enfermedad difusa y fue objeto de menos intervenciones por etapas. No obstante, no todas las características de este grupo fueron tan favorables puesto que la presencia de trombos y la lesión diana como la arteria asociada al infarto fueron más frecuentes en el grupo que SOLO recibió el stent Angiolite.

El objetivo primario, el FLD, al cabo de 1 año fue consistentemente bajo tanto en la población que SOLO recibió el stent Angiolite (análisis primario), 2.3\%, como en toda la población (análisis secundario), $2.8 \%$. El fracaso del vaso diana, una variable de seguridad más amplia, también fue visiblemente bajo $(3,1$ y $3,3 \%$, respectivamente). Para confirmar estos resultados, los MACE (incluida, también, la mortalidad por cualquier causa), una variable orientada clínicamente, también fue muy baja $(5,3$ y $6,3 \%$, respectivamente). La tabla 1 del material adicional ofrece una revisión de los resultados del FLD en los diferentes stents analizados en los distintos registros y ECA. En 

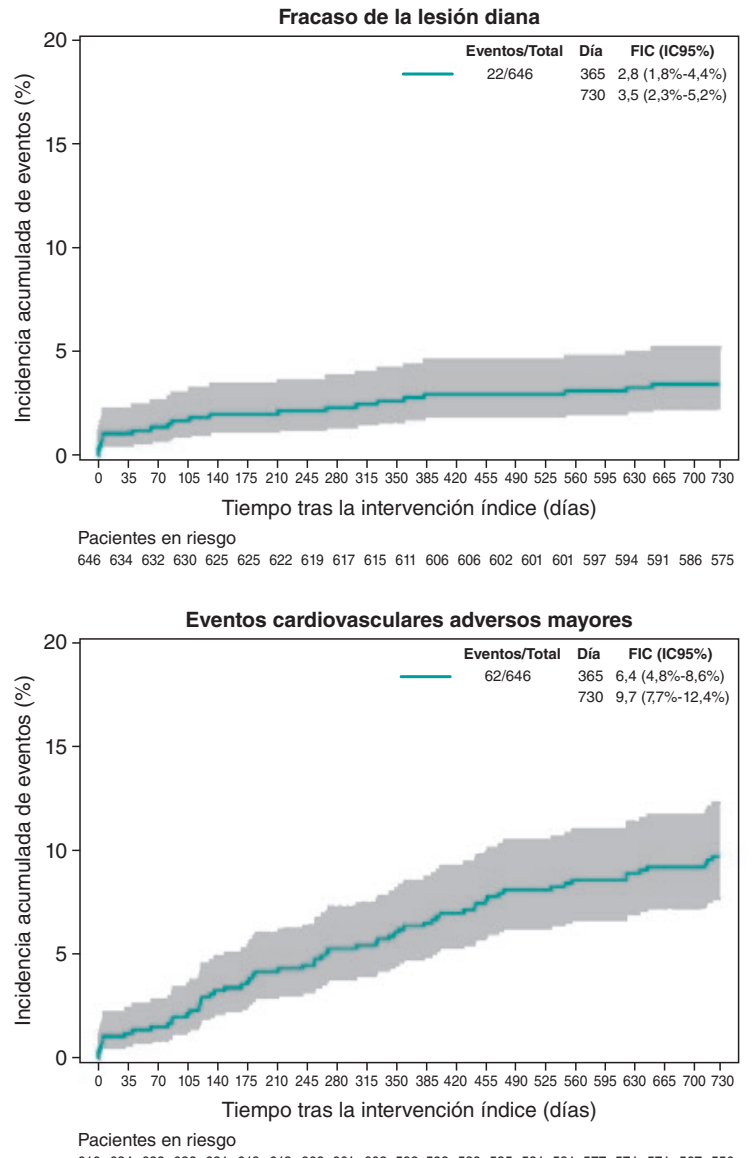
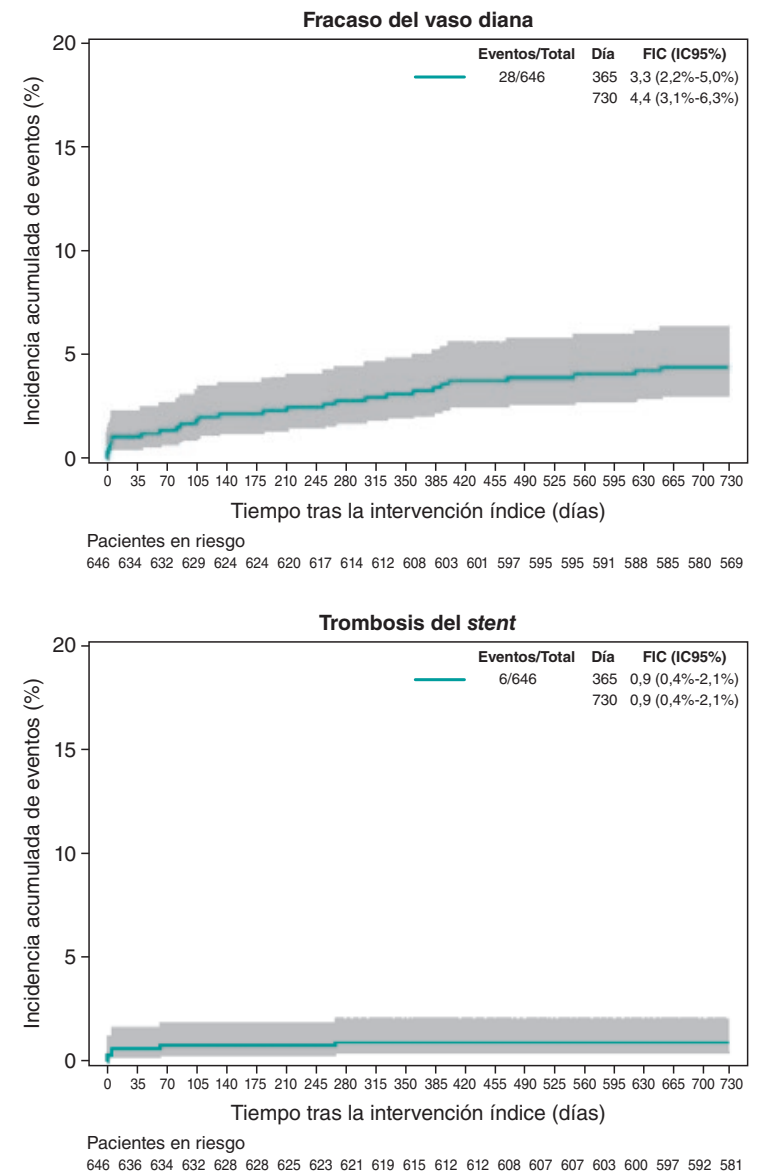

Figura 2. Incidencia acumulada a los 2 años de eventos en toda la población ( $n=646$ ).

estos estudios, el valor medio del FLD al cabo de 1 año es un 5,4\%, más alto que la incidencia descrita en este estudio.

El seguimiento a 2 años confirmó la incidencia sumamente baja de eventos cardiovasculares desfavorables durante el primer año. La incidencia de nuevos eventos cardiovasculares, orientados tanto al dispositivo como al paciente, durante el segundo año de seguimiento, fue la mitad de la incidencia observada durante el primer año.

Tanto el ANCHOR FIH ${ }^{5}$ como el ANGIOLITE RCT ${ }^{6}$ confirmaron la extraordinaria eficacia antiproliferativa del stent Angiolite con una pérdida luminal tardía media $<0,05 \mathrm{~mm}$. En consecuencia, nos pareció obligatorio valorar el perfil de seguridad de este stent a través de la incidencia de trombosis del stent. El uso del stent Angiolite en el mundo real se asoció a una incidencia baja de esta complicación tan catastrófica (del 0,7 en el análisis primario frente a $0,9 \%$ en el secundario), lo que garantiza el uso seguro de este potente SFA. Los estudios publicados han revelado una tasa de incidencia media de trombosis del stent del 0,4 al 4,9\% a los 2 años (tabla 1 del material adicional). La incidencia sumamente baja de trombosis del stent probable/definitiva más allá de la primera semana (solo 2 casos, 1 durante el primer mes y el otro, 268 días después) viene a revalidar el perfil de seguridad de este dispositivo. Se debe mencionar que el uso del tratamiento antiplaquetario doble fue alto en esta población (del $59,6 \%$ tras 1 año de seguimiento), lo cual sugiere la prevalencia del síndrome coronario agudo como presentación clínica de los pacientes (el 68,3\% de estos).

El análisis de subgrupos predefinidos dio resultados interesantes. Los pacientes diabéticos tuvieron una incidencia de FLD y
Tabla 5. Resultados del análisis poblacional primario: pacientes tratados solamente con el stent Angiolite

\begin{tabular}{llll}
\hline Solo población Angiolite $\mathbf{( n = 4 2 6 )}$ & $\begin{array}{l}\mathbf{6} \text { meses de } \\
\text { seguimiento }\end{array}$ & $\begin{array}{l}\mathbf{1} \text { año de } \\
\text { seguimiento }\end{array}$ & $\begin{array}{l}\mathbf{2} \text { años de } \\
\text { seguimiento }\end{array}$ \\
\hline Mortalidad & $5(1,2 \%)$ & $10(2,3 \%)$ & $18(4,2 \%)$ \\
\hline Mortalidad cardiovascular & $3(0,7 \%)$ & $5(1,2 \%)$ & $7(1,6 \%)$ \\
\hline Infarto de miocardio & $5(1,2 \%)$ & $5(1,2 \%)$ & $10(2,3 \%)$ \\
\hline $\begin{array}{l}\text { Infarto de miocardio del vaso diana } \\
\text { Trombosis definitiva/probable }\end{array}$ & $4(0,9 \%)$ & $4(0,9 \%)$ & $4(0,9 \%)$ \\
\hline del dispositivo & $3(0,7 \%)$ & $3(0,7 \%)$ & $3(0,7 \%)$ \\
\hline Revascularización & $7(1,6 \%)$ & $11(2,7 \%)$ & $18(4,2 \%)$ \\
\hline $\begin{array}{l}\text { Revascularización de la lesión } \\
\text { diana }\end{array}$ & $3(0,7 \%)$ & $4(0,9 \%)$ & $5(1,2 \%)$ \\
\hline Revascularización del vaso diana & $4(0,9 \%)$ & $7(1,6 \%)$ & $9(2,1 \%)$ \\
\hline $\begin{array}{l}\text { Revascularización del vaso } \\
\text { no diana }\end{array}$ & $3(0,7 \%)$ & $4(0,9 \%)$ & $9(2,1 \%)$ \\
\hline Fracaso de la lesión diana & $7(1,6 \%)$ & $10(2,3 \%)$ & $13(3,1 \%)$ \\
\hline Fracaso del vaso diana ${ }^{b}$ & $8(1,9 \%)$ & $13(3,1 \%)$ & $17(4,0 \%)$ \\
\hline MACE ${ }^{c}$ & $13(3,2 \%)$ & $22(5,3 \%)$ & $34(8,0 \%)$ \\
\hline
\end{tabular}

MACE: eventos cardiovasculares adversos mayores.

${ }^{a}$ Fracaso de la lesión diana definido como muerte cardiovascular, infarto de miocardio del vaso diana y revascularización de la lesión diana por motivos clínicos.

${ }^{b}$ Fracaso del vaso diana definido como muerte cardiovascular, infarto de miocardio del vaso diana y revascularización del vaso diana.

c MACE definidos como mortalidad por cualquier causa, cualquier infarto y cualquier revascularización. 

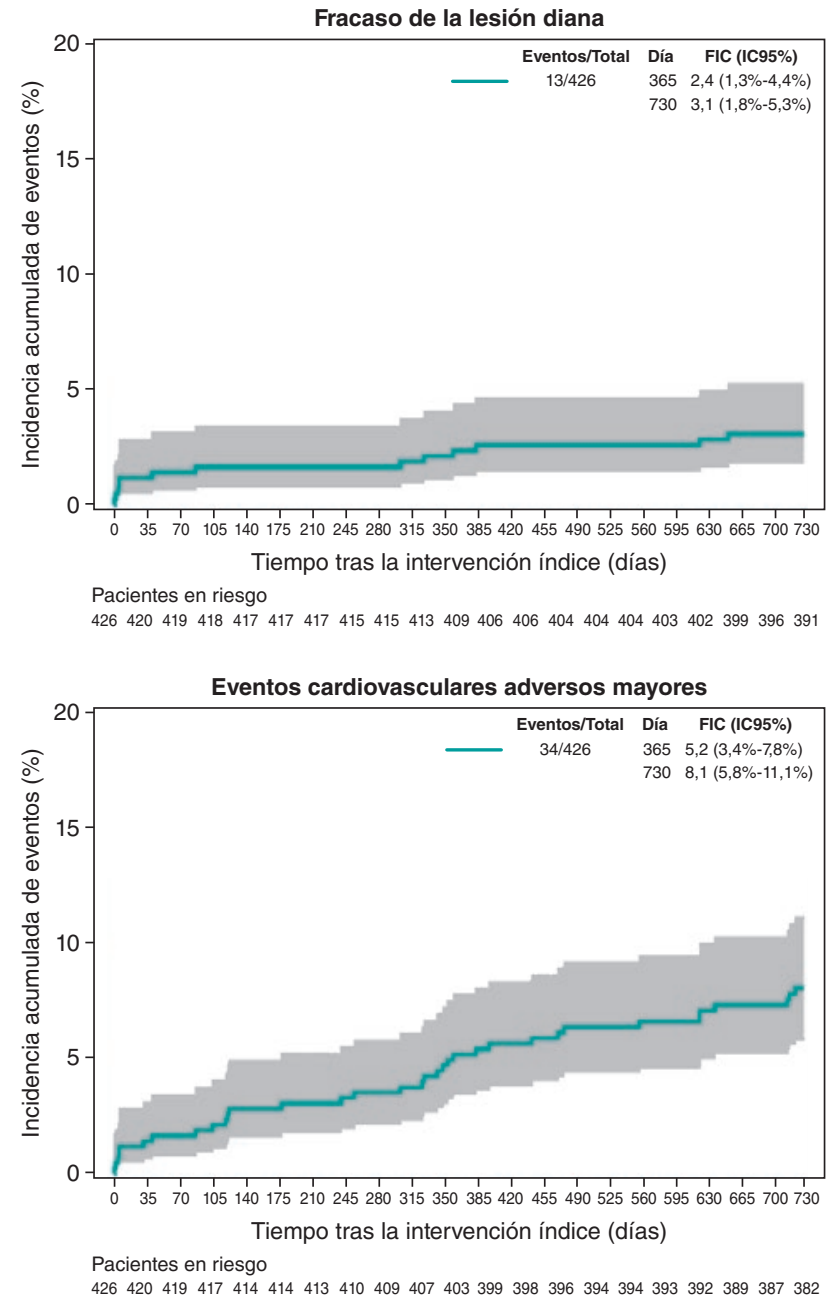

Fracaso del vaso diana

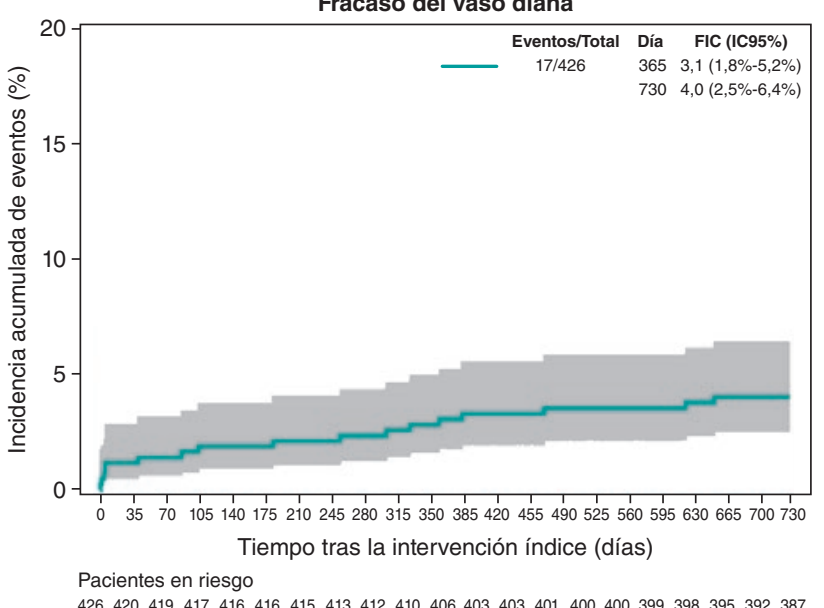

Trombosis del stent

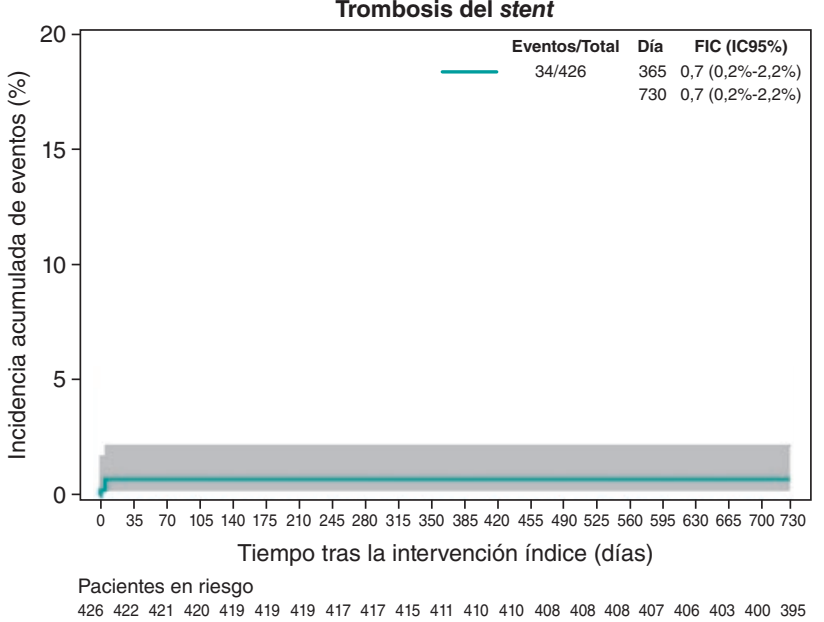

Figura 3. Incidencia acumulada a los 2 años de eventos en el análisis poblacional primario de pacientes tratados, solo, con el stent Angiolite (n = 426).

trombosis del stent a los 2 años similar a la tasa de incidencia global $(3,0$ frente a $3,4 \%$ y 1,0 frente a $0,9 \%$, respectivamente), si bien la incidencia de MACE fue más alta $(14,1$ frente a 9,6\%). Este hallazgo podría ser indicativo del peor pronóstico clínico que tienen los pacientes diabéticos y no estar necesariamente asociado a la lesión tratada, sino a la enfermedad coronaria residual. Nuestros resultados coinciden con los datos publicados con anterioridad en el subestudio EVOLVE II sobre diabetes ${ }^{13}$ que revelaron una incidencia de FLD a los 2 años del 11,2\% y una incidencia de trombosis del stent definitiva/probable del $1,1 \%$.

Como era de esperar, el subgrupo con enfermedad de pequeños vasos $(\leq 2,5 \mathrm{~mm})$ tuvo incidencias algo más altas de FLD y MACE a los 2 años $(4,3$ y $12,1 \%$, respectivamente) que las de la población global $(3,4$ y 9,6\%, respectivamente). La ausencia de casos con trombosis del stent definitiva/probable puede ser indicativo de sesgo de detección pues la expresión clínica de la trombosis de estos vasos podría ser menos evidente. Los resultados de este subgrupo suelen ser difíciles de comparar con otros datos porque la definición de vaso pequeño es sumamente arbitraria, de 2,25 a 3,0 mm. No obstante, los resultados de nuestro estudio coinciden con los descritos en el estudio Basket-Small ${ }^{18}$.

\section{Limitaciones}

Las limitaciones de este estudio son los ya conocidos problemas que presentan los registros observacionales del mundo real: posible sesgo de selección, sesgo de divulgación de los resultados y pérdidas durante el seguimiento (aunque no en el caso que nos ocupa pues se completó el $98 \%$ del periodo de seguimiento). No obstante, los resultados se parecen a los descritos anteriormente y coinciden con los resultados de anteriores estudios realizados con este stent. En la población global (pacientes que recibieron otros stents además de stents Angiolite), objetivos como la trombosis probable del stents o la muerte cardiovacular no se pueden atribuir directamente a un determinado tipo de stent. Para minimizar posibles errores y reforzar el mensaje de seguridad, el comité de dirección ha decido ampliar el periodo de seguimiento a un plazo de 5 años.

\section{CONCLUSIONES}

Los resultados de este registro observacional sobre el uso del SFA Angiolite en una población del mundo real confirman el excelente perfil de seguridad y eficacia de este dispositivo ya descrito en anteriores estudios tras 2 años de seguimiento. Se ha programado un seguimiento ampliado de 5 años de duración para descartar la ocurrencia eventos tardíos.

\section{FINANCIACIÓN}

La gestión de los datos y su análisis se han llevado a cabo por una organización de investigación clínica independiente. Tanto el borrador final como la redacción del manuscrito han corrido a cargo de investigadores, sin ninguna participación de los patrocinadores. 


\section{CONTRIBUCIÓN DE LOS AUTORES}

Idea y diseño: A. Pérez de Prado, F. Lozano Ruiz-Poveda, J. Moreu Burgos, B. García del Blanco, E. Pinar, V. Peral, J.R. Rumoroso y R. Trillo Nouche. Adquisición de los datos: A. Pérez de Prado, R. Ocaranza-Sánchez, F. Lozano Ruiz-Poveda, J. Moreu Burgos, R. Álvarez Ramos, A. Rodrigues, L. Fernández González, P. Aguar, B. García del Blanco, E. Pinar, V. Peral, F. Sainz Laso, J.R. Rumoroso, A. Torres, M. Sabaté y R. Trillo Nouche. Análisis estadístico y redacción del manuscrito: A. Pérez de Prado, F. Lozano RuizPoveda, J.R. Rumoroso y R. Trillo Nouche. Provisión de comentarios críticos al manuscrito y aprobación del contenido final: A. Pérez de Prado, R. Ocaranza-Sánchez, F. Lozano Ruiz-Poveda, J. Moreu Burgos, R. Álvarez Ramos, A. Rodrigues, L. Fernández González, P. Aguar, B. García del Blanco, E. Pinar, V. Peral, F. Sainz Laso, J.R. Rumoroso, A. Torres, M. Sabaté y R. Trillo Nouche.

\section{CONFLICTO DE INTERESES}

A. Pérez de Prado y M. Sabaté declararon haber recibido honorarios por su labor como consultores, así como becas de investigación de iVascular y Cardiva S.L. Por su parte, F. Lozano Ruiz-Poveda declaró haber recibido honorarios por su labor como conferenciante para Abbott y Medtronic. Todos los autores han declarado pagos a sus respectivos centros de parte de Cardiva S.L.

\section{¿QUÉ SE SABE DEL TEMA?}

- Los actuales SFA tienen una mayor eficacia a la hora de reducir la reestenosis y una incidencia muy baja de trombosis del stent. El stent Angiolite (iVascular, Barcelona, España) es un stent liberador de sirolimus fabricado en una aleación de cromo-cobalto y struts finos con un recubrimiento bioestable de poli-acrilato fluorado tromboresistente cargado con sirolimus. Este stent ha sido sometido a pruebas completas en estudios preclínicos, en el primer estudio (ANCHOR) realizado en humanos, así como en un ensayo clínico aleatorizado (que lo comparó con un stent de cromo-cobalto liberador de everolimus) con consistentes resultados positivos. Se diseñó un registro observacional y prospectivo que avalara los anteriores resultados descritos en nuestra práctica clínica habitual.

\section{¿QUÉ APORTA DE NUEVO?}

- Los resultados de este registro observacional sobre el uso del stent Angiolite en una población de alto riesgo del mundo real confirma los excelentes resultados de estudios ya publicados tras 2 años de seguimiento. Tanto el índice de resultados asociados al dispositivo (fracaso de la lesión y vaso dianas) como los resultados asociados al paciente (MACE) fueron más bajos comparados con datos anteriores.

\section{MATERIAL ADICIONAL}

Se puede consultar material adicional a este artículo en su versión electrónica disponible en https://doi.org/10.24875/ RECICE.M21000221.

\section{BIBLIOGRAFÍA}

1. Neumann F-J, Sousa-Uva M, Ahlsson A, Alfonso F, Banning AP, Benedetto U, et al. 2018 ESC/EACTS Guidelines on myocardial revascularization. European Heart Journal. 2018;40:87-165.
2. Lüscher TF, Steffel J, Eberli FR, Joner M, Nakazawa G, Tanner FC, et al. Drug-eluting stent and coronary thrombosis: biological mechanisms and clinical implications. Circulation. 2007;115:1051-8.

3. Gori T, Polimeni A, Indolfi C, Räber L, Adriaenssens T, Münzel T. Predictors of stent thrombosis and their implications for clinical practice. Nature Reviews Cardiology. 2019;16:243-256.

4. Estevez-Loureiro R, Perez de Prado A, Perez-Martinez C, Cuellas-Ramon C, Regueiro-Purrinos M, Gonzalo-Orden JM, et al. Safety and Efficacy of New Sirolimus-eluting stent Models in a Preclinical Study. Rev Esp Cardiol (Engl Ed). 2015;68:1118-24.

5. Puri R, Otaegui I, Sabate M, Serra-Penaranda A, Puigfel M, Perez de Prado A, et al. Three- and 6-month optical coherence tomographic surveillance following percutaneous coronary intervention with the Angiolite(R) drug eluting stent: The ANCHOR study. Catheter Cardiovasc Interv. 2018; 91:435-443.

6. Moreu J, Moreno-Gomez R, Perez de Prado A, Garcia Del Blanco B, Trillo R, Pinar E, et al. First-in-man randomised comparison of the Angiolite durable fluoroacrylate polymer-based sirolimus-eluting stent versus a durable fluoropolymer-based everolimus-eluting stent in patients with coronary artery disease: the ANGIOLITE trial. EuroIntervention. 2019;15:e1081-e1089.

7. Valgimigli M, Bueno H, Byrne RA, Collet J-P, Costa F, Jeppsson A, et al. 2017 ESC focused update on dual antiplatelet therapy in coronary artery disease developed in collaboration with EACTS: The Task Force for dual antiplatelet therapy in coronary artery disease of the European Society of Cardiology (ESC) and of the European Association for Cardio-Thoracic Surgery (EACTS). European Heart Journal. 2017;39:213-260.

8. Garcia-Garcia HM, McFadden EP, Farb A, Mehran R, Stone GW, Spertus $\mathrm{J}$, et al. Standardized End Point Definitions for Coronary Intervention Trials: The Academic Research Consortium-2 Consensus Document. Circulation. 2018;137:2635-2650.

9. Stone GW, Rizvi A, Sudhir K, Newman W, Applegate RJ, Cannon LA, et al. Randomized comparison of everolimus- and paclitaxel-eluting stents. 2-year follow-up from the SPIRIT (Clinical Evaluation of the XIENCE V Everolimus Eluting Coronary stent System) IV trial. J Am Coll Cardiol. 2011;58:19-25.

10. Waltenberger J, Hoffmann S, Brachmann J, Van Der Heijden J, Richardt G, Froebert O, et al. Bioflow-III: one year target lesion failure data of an all-comers registry with a drug eluting stent. European Heart Journal. 2013;34:P3036-P3036.

11. Pilgrim T, Heg D, Roffi M, Tüller D, Muller O, Vuilliomenet A, et al. Ultrathin strut biodegradable polymer sirolimus-eluting stent versus durable polymer everolimus-eluting stent for percutaneous coronary revascularisation (BIOSCIENCE): a randomised, single-blind, non-inferiority trial. Lancet. 2014;384:2111-22.

12. Kereiakes DJ, Meredith IT, Windecker S, Lee Jobe R, Mehta SR, Sarembock IJ, et al. Efficacy and safety of a novel bioabsorbable polymer-coated everolimus-eluting coronary stent : the EVOLVE II Randomized Trial. Circ Cardiovasc Interv. 2015;8

13. Kereiakes DJ, Meredith IT, Masotti M, Carrie D, Moreno R, Erglis A, et al. Safety and efficacy of a bioabsorbable polymer-coated, everolimus-eluting coronary stent in patients with diabetes: the EVOLVE II diabetes substudy. EuroIntervention. 2017;12:1987-1994.

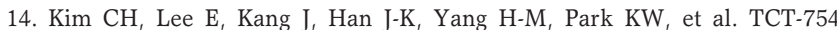
One-year clinical outcome of patients treated with Resolute Onyx versus Resolute Integrity: A Comparison of the HOST-ONYX and HOST-RESOLINTE Registries. Journal of the American College of Cardiology. 2017 70:B319-B319.

15. Tam CC, Chan K, Lam S, Yung A, Lam YM, Chan C, et al. One-year clinical outcomes of patients implanted with a Resolute Onyx zotarolimus-eluting stent. J Int Med Res. 2018;46:457-463.

16. von Birgelen C, Zocca P, Buiten RA, Jessurun GAJ, Schotborgh CE, Roguin A, et al. Thin composite wire strut, durable polymer-coated (Resolute Onyx) versus ultrathin cobalt\&\#x2013; chromium strut, bioresorbable polymercoated (Orsiro) drug-eluting stents in allcomers with coronary artery disease (BIONYX): an international, single-blind, randomised non-inferiority trial. The Lancet. 2018;392:1235-1245.

17. Wijns W, Valdes-Chavarri M, Richardt G, Moreno R, Iniguez-Romo A, Barbato $\mathrm{E}$, et al. Long-term clinical outcomes after bioresorbable and permanent polymer drug-eluting stent implantation: final five-year results of the CENTURY II randomised clinical trial. EuroIntervention. 2018;14: e343-e351.

18. Jeger RV, Farah A, Ohlow M-A, Mangner N, Möbius-Winkler S, Leibundgut $G$, et al. Drug-coated balloons for small coronary artery disease (BASKETSMALL 2): an open-label randomised non-inferiority trial. The Lancet. 2018;392:849-856 